\title{
BMJ Open The REAnimation Low Immune Status Markers (REALISM) project: a protocol for broad characterisation and follow-up of injury-induced immunosuppression in intensive care unit (ICU) critically ill patients
}

Mary-Luz Rol, ${ }^{1,2}$ Fabienne Venet, ${ }^{2,3}$ Thomas Rimmele, ${ }^{2,4}$ Virginie Moucadel, ${ }^{5}$ Pierre Cortez, ${ }^{6}$ Laurence Quemeneur, ${ }^{7}$ David Gardiner, ${ }^{13}$ Andrew Griffiths, ${ }^{8}$ Alexandre Pachot, ${ }^{2,5}$ Julien Textoris, ${ }^{2,4,5}$ Guillaume Monneret, ${ }^{2,3}$ On behalf of the REALISM study group

To cite: Rol M-L, Venet $F$, Rimmele T, et al. The REAnimation Low Immune Status Markers (REALISM) project: a protocol for broad characterisation and follow-up of injury-induced immunosuppression in intensive care unit (ICU) critically ill patients. BMJ Open 2017;7:e015734. doi:10.1136/ bmjopen-2016-015734

- Prepublication history and additional material are available. To view these files please visit the journal online (http://dx.doi.org/ 10.1136/ bmjopen-2016-015734).

Received 2 January 2017 Revised 22 February 2017 Accepted 8 March 2017

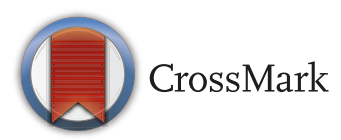

For numbered affiliations see end of article.

Correspondence to Dr Julien Textoris; julien. textoris@biomerieux.com

\begin{abstract}
Introduction The host response to septic shock is dynamic and complex. A sepsis-induced immunosuppression phase has recently been acknowledged and linked to bad outcomes and increased healthcare costs. Moreover, a marked suppression of the immune response has also been partially described in patients hospitalized in intensive care unit (ICU) for severe trauma or burns. It has been hypothesized that immune monitoring could enable identification of patients who might most benefit from novel, adjunctive immune-stimulating therapies. However, there is currently neither a clear definition for such injury-induced immunosuppression nor a stratification biomarker compatible with clinical constraints.

Methods and analysis We set up a prospective, longitudinal single-centre clinical study to determine the incidence, severity and persistency of innate and adaptive immune alterations in ICU patients. We optimized a

\section{Strengths and limitations of this study}

- This is the first prospective study to provide a broad immune status characterisation in a large cohort of intensive care unit (ICU) patients.

- There is a mid-term assessment (D60) of the immune status in ICU patients, which has never been done before.

- Long-term follow-up will not be addressed here and should be examined in future studies.

- New biomarkers of the immune status will be assessed in comparison to standardised tools and immune functional assays.

- Whether such biomarkers would permit to stratify patients for immunomodulatory treatments should be addressed in future studies.

- The role of host genomics, microbiota as well as checkpoint inhibitor expressions will not be assessed in this study.
\end{abstract} workflow to describe and follow the immunoinflammatory status of 550 patients (septic shock, severe trauma/burn and major surgery) during the first 2 months after their initial injury. On each time point, two immune functional tests will be performed to determine whole-blood TNF- $\alpha$ production in response to ex vivo lipopolysaccharide stimulation and the T lymphocyte proliferation in response to phytohaemagglutinin. In addition, a complete immunophenotyping using flow cytometry including monocyte HLA-DR expression and lymphocyte subsets will be obtained. New markers (ie, levels of expression of host mRNA and viral reactivation) will be also evaluated. Reference intervals will be determined from a cohort of 150 age-matched healthy volunteers. This clinical study will provide, for the first time, data describing the immune status of severe ICU patients over time.

Ethics and dissemination Ethical approval has been obtained from the institutional review board (no 69HCL15_0379) and the French National Security agency for drugs and health-related products. Results will be disseminated through presentations at scientific meetings and publications in peer-reviewed journals.

Trial registration number Clinicaltrials.gov Registration number: NCT02638779. Pre-results.

\section{INTRODUCTION}

Sepsis is a major health problem and the main aetiology for intensive care unit (ICU) admissions. ${ }^{12}$ Its incidence is increasing over the years due to several factors, including a better awareness and an ageing population. ${ }^{3}$ Hospital admissions for sepsis have thus overtaken those for stroke and myocardial infarction. ${ }^{4}$ Despite advances on its management, mortality of sepsis has remained stable over the last 20 years, reaching 30\%-40\% in 
case of septic shock, the most severe form, and it is the leading cause of death in ICU.

Sepsis is a severe infection, defined as a 'life-threatening organ dysfunction caused by a dysregulated host response to infection' ${ }^{5}$ Besides circulatory and metabolic abnormalities, the multifaceted host response to the invading pathogen is amplified by comorbid conditions. ${ }^{67}$ It is now acknowledged that the pro-inflammatory response, which can lead to organ failure, comes with a compensatory anti-inflammatory response. Recovery occurs when inflammation resolves quickly. However, in numerous patients, the anti-inflammatory response lingers on and leads to an immunosuppression state, associated with secondary infections, and increased morbidity and mortality. ${ }^{8}$ This sepsis-induced immunosuppression could explain the failure of several previous clinical trials and support new innovative trials testing immune adjuvant drugs in septic shock. $^{9}$

Therefore, several studies and case reports now support the rational of boosting the immune system, in order to avoid the occurrence of healthcare-associated infection and therefore reduce the associated morbidity. ${ }^{10} 11$ However, to avoid reproducing the errors from the past, such innovative treatments should be administered only to those individuals identified as immunosuppressed. ${ }^{11}$ Some studies have already demonstrated that the concept of biomarker-guided therapeutic stratification can lead to clinical improvements. ${ }^{12}$

A marked immunosuppression has been partially described in other patients admitted to the ICU for severe trauma/burns and other major surgeries. ${ }^{13-16}$ In these 'sterile' injuries, signs of injury-induced immune alterations have also been associated with increased susceptibility to secondary infections and mortality.

Given the complexity and heterogeneity of ICU patients, it is unlikely that any single biomarker will be sufficient to describe and diagnose injury-induced immunosuppression. On the contrary, a panel of validated biomarkers may bring enough information to accomplish such complex endeavour.

\section{Rationale of the study}

From a clinical perspective, no specific clinical signs or symptoms are associated with a state of altered immune response to allow prospective identification of at risk patients. Further, the outcomes of sustained immunosuppression are best defined by clinical relevant endpoints such as the occurrence of opportunistic and secondary infections. However, waiting for such a healthcare-associated infection to occur does not facilitate implementation of preventive strategies. Thus, diagnosis will rely on biomarkers.

From a biological perspective, sepsis-induced immunosuppression may be best identified by immune functional assays (such as cytokine release or lymphocyte proliferations after ex vivo stimulation) or by cell count parameters (such as number of lymphocytes or level of expression of mHLADR) but both approaches present drawbacks. Indeed, such functional assays are not suitable to stratify patients in a prospective interventional clinical trial due to (1) the long time to results (up to 5 days for lymphocytes proliferation) and (2) poor reproducibility due to standardisation issues and cumbersome technique. Due to such complexity, these reference tests are rarely performed in clinical studies evaluating biomarkers associated with deleterious outcomes in ICU.

On the other hand, HLA-DR expression on monocytes is currently the best biomarker available for such a routine use, ${ }^{17}$ and it is being employed for patient stratification in a large multicentre interventional trial assessing the administration of GM-CSF in patients with septic shock. ${ }^{18}$ However, its measurement requires flow cytometry analysis within 4 hours of blood sampling which may not be available in all centres, making interlaboratory standardisation challenging.

As a consequence of the previously discussed challenges, numerous biomarkers proposed to monitor injury-induced immune alterations have yet to be compared with these reference assays.

\section{Hypothesis}

Although several studies have shown an association between markers related to the immune system (eg, HLA-DR) and the occurrence of healthcare-associated infections in septic patients, ${ }^{14} 1519$ we still do not have a clear and operational definition of the immune deficiency that occurs in severely injured ICU patients. Precise description of injury-induced immunosuppression incidence and its characteristics are lacking. In the REALISM (REAnimation Low Immune Status Markers) project, we propose to broadly assess immune parameters over time and to correlate these findings with clinical epidemiological data and outcomes in order to identify and define immunosuppression in ICU patients in terms of both magnitude and time duration.

To this aim, we have established two standardised functional immune assays (whole-blood TNF- $\alpha$ release after ex vivo stimulation with LPS (lipopolysaccharides) ${ }^{20}$ and lymphocyte proliferation in response to ex vivo stimulation with PHA (phytohaemagglutinin). ${ }^{21}$ We propose to define the status of immunosuppression on the basis of an abnormal result (values outside the reference intervals) obtained in at least one of the two 'reference' tests.

The REALISM project aims to provide a validated operational definition of injury-induced immunosuppression predicting clinically relevant outcomes. This will facilitate development of new tools and biomarkers with the goal of introducing diagnosis of immune suppression into routine clinical practice and will allow patient stratification for the evaluation of new individual immunotherapies.

It may also enable the identification of new targets and the development of new innovative therapeutics to treat ICU patients and prevent opportunistic infections in the future 


\section{Primary aim}

The primary objective of the study is to determine the incidence of injury-induced immunosuppression in ICU patients, during the first 2 months after injury.

\section{Secondary aims}

The secondary objectives of the study are as follows:

- To describe the occurrence of immunosuppression, its depth and impact on innate and adaptive immune responses and its evolution during the first 2 months after injury.

- To assess the strength of the proposed definition, in particular, by evaluating its association with secondary infections and mortality.

- To assess the accuracy of new biomarkers and immune functional assays to diagnose immunosuppression.

These new biomarkers / immune functional assays could therefore replace assays such as the $\mathrm{T}$ cell proliferation assay, the current protocol of which is not suited to the routine management of ICU patients. We therefore expect to provide data to validate simpler diagnostic tools to determine and follow the immune status in hospitalised patients.

\section{METHODS AND ANALYSIS}

REALISM is a prospective longitudinal, single-centre observational study, conducted in the anaesthesiology and intensive care department at the Edouard Herriot Hospital (University Hospital, Lyon, France; capacity of approximately 1000 beds).

\section{Study population}

REALISM will include healthy volunteers $(\mathrm{n}=150)$ and patients at risk of injury-induced immunosuppression: (1) septic shock patients $(\mathrm{n}=160)$, (2) severe trauma patients $(n=180)$, (3) severe burns patients $(n=30)$ and (4) patients admitted to the ICU after major surgery $(n=180)$.

Septic shock inclusion criteria follow the current defini$\operatorname{tion}^{5}$ and require a state of shock defined by vasopressors administration and plasma lactate level above $2 \mathrm{mmol} / \mathrm{L}$ $(18 \mathrm{mg} / \mathrm{dL})$. An infection must be suspected, and microbiological sampling should have been performed, along with the administration of antimicrobials. Only primary septic shock will be considered (vasopressors should have been started within the first 48 hours after ICU admission).$^{5}$

Patients with severe trauma, defined by an ISS (injury severity score, Baker et al, 1974) $>15,{ }^{22}$ will be included in the study. As we hypothesised that the depth of

\section{Box 1 Inclusion and exclusion criteria for patients}

\section{Inclusion criteria}

Male or female aged over 18 years

Patient hospitalised for:

Septic shock, defined by:

Infection site suspected, and microbiological analysis sampling carried out

Vasopressor therapy needed to elevate mean arterial pressure $\geq 65 \mathrm{~mm} \mathrm{Hg}$ and lactate $>2 \mathrm{mmol} / \mathrm{L}$ (18 $\mathrm{mg} / \mathrm{dL})$ despite adequate fluid resuscitation ${ }^{27}$

Norepinephrine $>0.20 \mu \mathrm{g} / \mathrm{kg} / \mathrm{min}$ for at least 2 hours

Norepinephrine started within 48 hours after intensive care unit (ICU) admission

Serious trauma, defined by:

Patient admitted directly to the recruiting ICU

ISS, Baker et al, $1974>15^{22}$

Severe burns, defined by:

Total burned surface area $>30 \%$

Major surgery, defined by:

Surgery set for one of the following indications: (1) eso-gastrectomy, (2) Bricker's bladder resection (total bladder resection with reconstruction from small bowel), (3) cephalic pancreaticoduodenectomy (Whipple's procedure) and (4) abdominal aortic aneurysm surgery by laparotomy. Categories 1-3 concern management of solid tumours, while category 4 concerns non-cancerous pathologies

Induction of anaesthesia before 11:00 (to permit same-day processing of all samples)

\section{Exclusion criteria}

Patient with severe neutropenia (neutrophil count $<0.5 \mathrm{G} / \mathrm{L}$ )

Patients receiving immunosuppressive therapy

Corticosteroids (intravenously or per os).

Use of therapeutic antibodies

Onco-haematological disease (eg, lymphoma, leukaemia...) under treatment or treated within 5 years before inclusion

End of chemotherapy within the 6 months prior to inclusion date

Patient with innate or acquired immune deficiency (eg, severe combined immunodeficiency, HIV or AIDS, any stage)

Patients with a 'do not resuscitate order' or a 'withdraw of care' decision, at time of inclusion

Patient whose anticipated duration of hospitalisation in the ICU is estimated at less than 48 hours

Participation in any interventional study

Extra-corporeal circulation in the month preceding inclusion in the case of cardiac surgery

Pregnant or breastfeeding women

Patient with no social security insurance, with restricted liberty or under legal protection 
Box 2 Inclusion and exclusion criteria for healthy volunteers

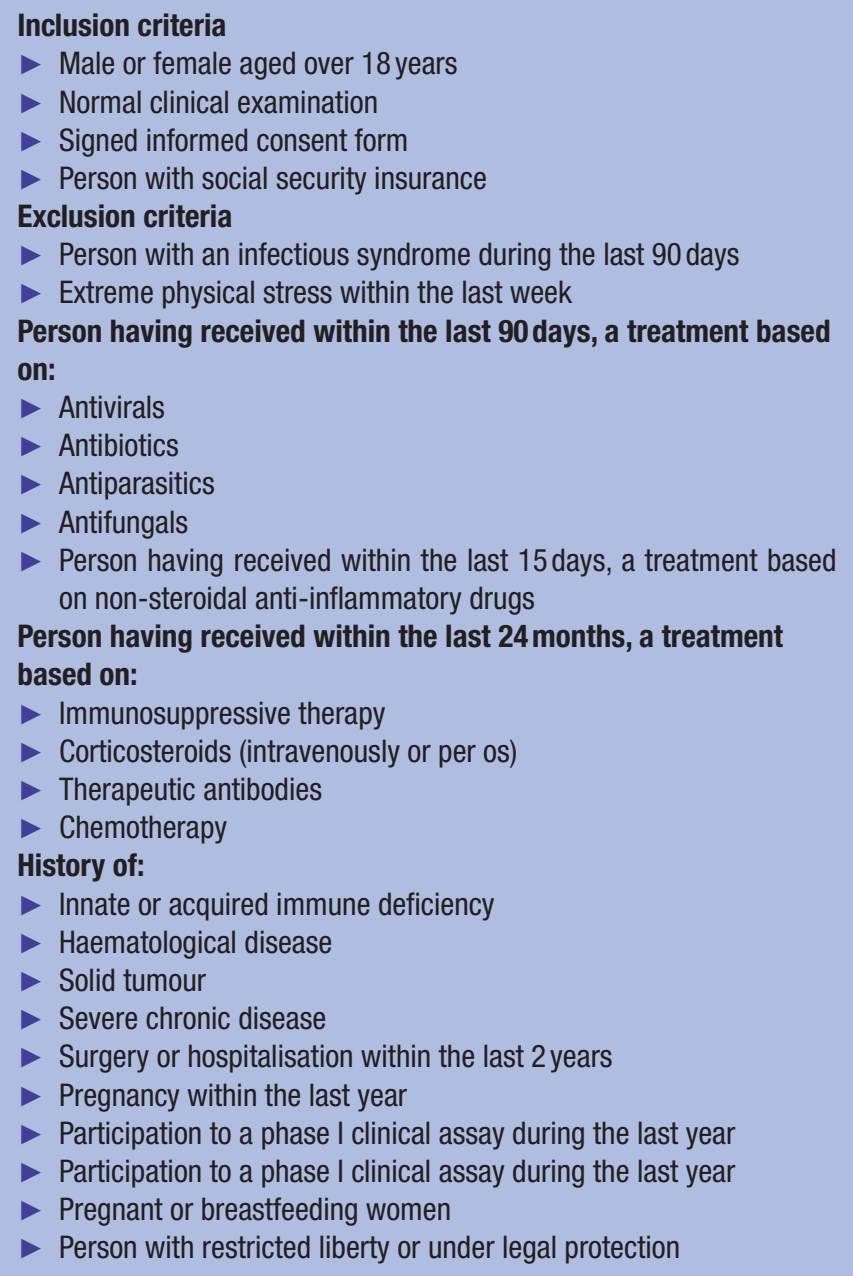

immunosuppression might be related to severity, we will limit the group of patients between $\operatorname{ISS}^{15-17}$ 19-26 values to 90 patients to ensure that, at least, $50 \%$ of the cohort includes patients with an ISS $>25$. Severe burn patients will be selected for inclusion based on a total burn surface area over $30 \%$.

Surgical patients will be screened according to the planned surgical procedure. This study will include patients undergoing: (1) eso-gastrectomy, (2) Bricker's bladder resection (total bladder resection with reconstruction from small bowel), (3) cephalic

Table 1 Age and gender distribution for the reference group

\begin{tabular}{lll}
\hline Age range & Male & Female \\
\hline$(19-30)$ & 14 & 14 \\
$(30-50)$ & 25 & 25 \\
$(50-65)$ & 18 & 19 \\
$(65-100)$ & 15 & 20 \\
Total & 72 & 78 \\
\hline
\end{tabular}

pancreaticoduodenectomy (Whipple's procedure) and (4) abdominal aortic aneurysm surgery by laparotomy.

Exclusion criteria are mainly related to factors that might impact the immune status and bias the results such as the following: severe neutropenia (neutrophil count $\left.<0.510^{9} / \mathrm{L}\right)$, administration of immunosuppressive therapy, corticosteroids (IV or oral administration), use of therapeutic antibodies (such as anti-TNF- $\alpha$ ), onco-haematological disease (eg, lymphoma, leukaemia) under treatment or treated within 5 years before inclusion and end of chemotherapy within the 6 months prior to inclusion date. Patients with congenital/hereditary or acquired immune deficiency (eg, severe combined immunodeficiency, HIV or AIDS, at any stage) and patients that have received extracorporeal circulation in the month preceding inclusion will be excluded as well.

Considering the possible influence of gender bias on measured parameters, we will recruit healthy donors from both genders, following the age and gender distribution of the French population.

Complete lists of the inclusion and exclusion criteria for patients and healthy volunteers are presented in box 1 and box 2, respectively.

\section{Sampling schedule}

Samples and clinical data will be collected 3-4 times within the first week (early time points) with the aim to evaluate the modulation of the immune status early after injury. Samples will be collected at day 1 (the morning following injury), at day 2 (for the severe trauma group) and at day $3 / 4$ and day $5 / 7$ (table 1 ). Samples will also be collected before surgery, at day 0 , as surgical patients are the only group for which sampling can be performed before injury. Additional samples will be collected during late time points to evaluate the recovery of the immune status, at day 14 (between day 13 and 18), day 28 (between day 26 and 36) and day 60 (between day 52 and 68), depending on patient availability and technical constraints (figure 1). Total volume of sampling will be $30 \mathrm{~mL}$ at each time point.

\section{Definition of immunosuppression}

The REALISM project will monitor the immune function of the patients and healthy volunteers using two standardised immune functional tests: one reference test to evaluate the innate immune response (wholeblood production of $\mathrm{TNF}-\alpha$ in response to ex vivo stimulation by LPS) and a second reference test for the adaptive immune response (the lymphocyte proliferation in response to ex vivo T cell stimulation with PHA). Immunosuppression will be defined in comparison to the values as obtained in a group of healthy volunteers for the two reference tests using the following methodology. First, reference intervals will be derived from the independent set of healthy volunteers. Second, immunosuppression will be defined in a patient when an abnormal result (value outside the reference intervals) 
is obtained in at least one of the two 'reference' tests over at least two consecutive time points

\section{Definition of secondary infection}

During the ICU stay, patients will be screened daily for exposure to invasive devices (intubation, indwelling urinary catheter and central venous line) and occurrence of secondary infection. Information referent to infections will be collected, reviewed and validated by a dedicated adjudication committee, composed of three physicians not involved in the recruitment of the patients with confirmation of secondary infection made according to the definitions used by the European Centre for Disease Prevention and Control $^{24}$ and the Infectious Diseases Society of America.

\section{Immune functional assays}

Innate immune response: TNF- $\alpha$ release after LPS whole-blood stimulation

Innate immune response will be evaluated by measuring the production of TNF- $\alpha$ in response to ex vivo stimulation of whole blood by LPS. ${ }^{20}$ The stimulation will be performed through the use of standardised TruCulture tubes from MYRIAD RBM (MYRIAD RBM, Austin, USA) (the concentration, quality and activity of the LPS is guaranteed by the manufacturer MYRIAD RBM). ${ }^{20}$ The tubes contain the medium alone (Null) or the medium with LPS $100 \mathrm{ng} / \mathrm{mL}$ (LPS from Escherichia coli O55:B5) (LPS-R; Null-R; MYRIAD RBM). The blood samples will be collected on heparin and transported to the laboratory where $1 \mathrm{~mL}$ of heparinized blood will be transferred to each TruCulture tube and incubated for 24 hours at $37^{\circ} \mathrm{C}$. Following incubation, the supernatant (medium+plasma) will be collected using a separation valve (according to manufacturer instructions) and stored at $-80^{\circ} \mathrm{C}$ until batch quantification of TNF- $\alpha$ by ELISA (BE55001; BL International-Tecan, Männedorf, Switzerland).

Adaptive immune response: T lymphocyte proliferation after ex vivo peripheral blood mononuclear cells mitogenic stimulation

Adaptive immune response will be assessed by measuring $\mathrm{T}$ lymphocyte proliferation in response to ex vivo stimulation with a mitogen. ${ }^{21}$ Briefly, peripheral blood mononuclear cells isolated by Ficoll density gradient centrifugation (U-04; Eurobio, Les Ulis, France) will be stimulated with PHA at $4 \mu \mathrm{g} / \mathrm{mL}$ (HA16; Remel, Lenexa, USA), at $37^{\circ} \mathrm{C}$ for 72 hours. Following incubation, the cells will be harvested and cell's proliferation will be determined by the incorporation of EdU (5-ethynyl-2'-deoxyuridine, $10 \mu \mathrm{M}$ for 2 hours) in T cells using the commercial kit Click-It EdU AF488 flow kit (C10420; Life Technologies, Carlsbad, California, USA). Cell proliferation is measured as the percentage of EdU-positive T cells (gated as CD3+ using a CD3-APC staining) using flow cytometry. ${ }^{21}$

\section{Cellular immunophenotyping}

Complete blood cell count report from the haematology laboratory will be collected on each time point, this information will be compared with our cell counts results by flow cytometry. Beside phenotypic immune cells, characterisation and cell counting will be completed by flow cytometry and we will count the number of B lymphocytes (CD45+, CD3-, CD19+), T lymphocytes, CD4+ (CD45+, CD3+, CD8-, CD4+) and CD8+ (CD45+, CD3+, CD8+, CD4-), NK cells (CD45+, CD3-, CD56+), regulatory $\mathrm{T}$ lymphocytes (gated on $\mathrm{T}$ CD4+, CD25high, CD127low) and mature (CD10High, CD16High, CD14-, CRTH2-) and immature mature (CD10dim, CD16dim, CD14-, CRTH2-) polymorphonuclear cells, as previously published. ${ }^{25}{ }^{26}$ In addition, the number of HLA-DR molecules per monocyte will be determined using the BD quantibrite standardised method (HLA-DR:340827; QuantiBRITE:340495; Becton Dickenson, New Jersey, USA) ${ }^{27}$ It is well known that the flow cytometry is highly sensitive to variation between laboratories and instruments; therefore, a validation with the routine hospital immunology laboratory was performed to guarantee that all the protocols are reproducible and standardised. All procedures generated results with less than $20 \%$ of variation when compared with reference protocols.

\section{Biobanking}

This study will provide the opportunity to establish four different types of biobanks to preserve the material collected, enabling exploration of innovative biomarkers:

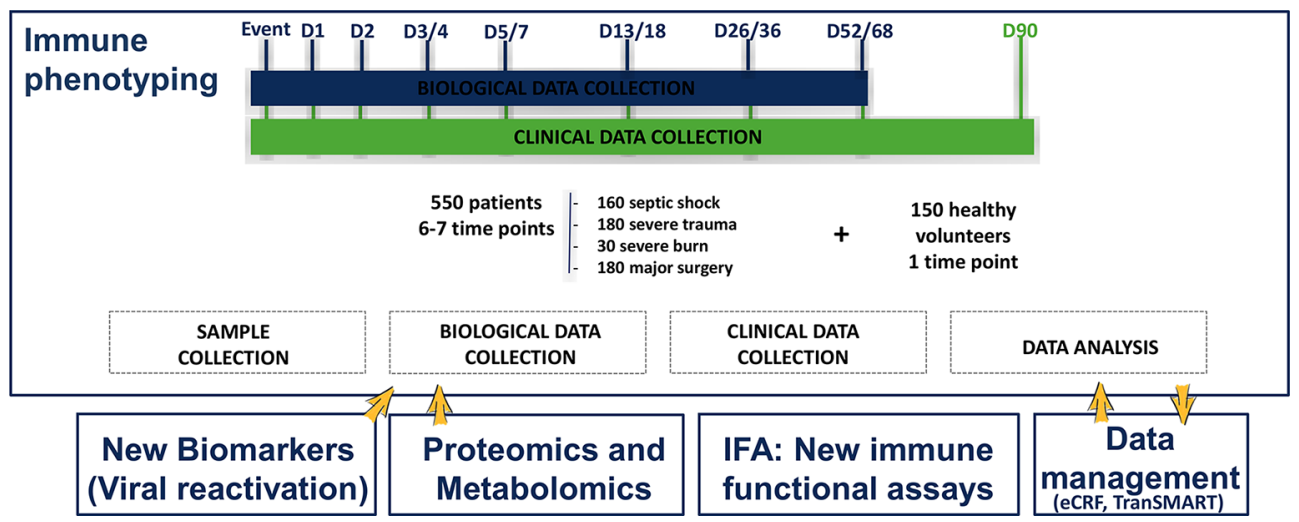

Figure 1 Schematic design of the REALISM project illustrating the type of patients included in the study, the various time points and major planned analysis. REALISM, REAnimation Low Immune Status Markers. 
(1) TruCulture plasma biobank from whole blood stimulated with LPS, SEB (Staphylococcus aureus enterotoxin B) or not stimulated, to study cytokines release; (2) EDTA plasma biobank to study viral reactivation markers and soluble host biomarkers; (3) heparin plasma biobank for metabolomics/proteomics soluble host biomarkers studies and (4) RNA biobank to study new transcriptomic host biomarkers (RNA will be extracted from whole blood collected in PAXgene tubes).

\section{Innovative immune functional assays and exploration of new biomarkers}

Regarding the immune functional tests, other stimulants (eg, SEB) and read-outs (eg, interleukin 2, interferon gamma) will be tested using the TruCulture tubes. The cytokine production levels in the supernatants of the functional assays will be quantified using commercial IVD or RUO assays. Finally, a metabolomics and proteomics study will be performed using frozen (heparin) plasma. Biomarkers potentially associated to immune deficiency will be identified by liquid chromatography-mass spectrometry on high-resolution mass spectrometry and ${ }^{1} \mathrm{H}$ nuclear magnetic resonance, after polar and non-polar samples extraction.

\section{Sample size and data analysis plan Population sizing}

The number of healthy volunteers required to determine the reference intervals for the two immune reference tests was defined according to the methodology recommended by the Clinical and Laboratory Standards Institute C28-A3 guidelines. ${ }^{28}$ The minimal number of subjects recommended being 120, after exclusion of aberrant values (CI of $90 \%$ ), we decided to include 150 healthy volunteers to take into account exclusions related to technical reasons, aberrant values or consent withdrawal.

For this reference population, the age range of healthy volunteers group has been carefully calculated to include the expected age range and gender distribution from ICU patients in France (table 1).

The main objective being descriptive, the computation of the sample size was based on secondary objectives, especially for (1) the analysis of the occurrence of immunosuppression, its depth and impact on innate and adaptive immune responses (Cohen's $d$ is 0.55 ) and (2) the correlation between new biomarkers and immune functional assays to diagnose immunosuppression $(r>0.4)$. A Student's t-test was used to approximate the number of patients needed and a minimum of 150 patients per group was required for a standardised Cohen's d effect $=0.55$, if we get the recommended number of healthy volunteers of 120. It was therefore decided to include 160 septic shock patients, 180 severe trauma patients and 180 patients with a major surgery, to overcome secondary exclusions for technical causes or consent withdrawal. The severe burn patients group is an ancillary group that was arbitrary fixed at 30 subjects in order to collect data with the intent to inform a dedicated study on this population in the future.

\section{Statistical analysis}

First, the percentage of patients meeting the definition of injury-induced immunosuppression will be computed in each patients group to answer the main objective. Second, the occurrence of immunosuppression will be further described. The proportion of patients with at least one abnormal test will be computed for both immune reference tests and each patients group. The correlation between the two reference tests will be established from a Spearman correlation test. A mixed model will be constructed to describe the extent of the changes in the innate and adaptive measures over time, taking groups and time points into account. Third, a comparison of each biomarker or new functional tests with the two reference tests will be performed using a Spearman correlation test. For correlated biomarkers or functional tests, the performance for prediction of secondary infection will be estimated from a receiver operating characteristic curve. A Fine \& Grey predictive model will be constructed ${ }^{29}$ for the biomarkers harbouring the best areas under curve, taking into account the competing risk of mortality. Finally, multiple imputations will be taken into consideration in the case of a relevant amount of missing values.

\section{ETHICS AND DISSEMINATION \\ Ethics approval}

The protocol, information documents and consent forms received approval by the local institutional review board (Comité de Protection des Personnes Sud-Est II, Bron, France) and the French National Security agency for drugs and health-related products (Approval code: 69HCL15_0379, 30November 2015). An amendment has been filled to extend sampling time points over the first week and add the metabolomics and proteomics study. This amendment has been approved on the 22July 2016 (protocol version 3). This study complies with the Declaration of Helsinki, principles of Good Clinical Practice and the French personal data protection act.

\section{Informed consent}

The free and informed consent of each patient and healthy volunteer will be obtained following a complete and faithful information, in comprehensive words, of the objectives, the proceedings and the constrains of the study, the right to refuse the enrollment or the possibility to withdraw at any time, when he/she is in capacity to understand. The patient (or next of kin) will also be informed of (1) the existence of processing system for data concerning them, (2) Their right to access and rectify these data (accessible through the physician of their choice) and (3) the possibility of the use of remaining biological material and associated data stored following the end of the study and their possible transfer to another academic or private party. This information is part of the written notice and the informed consent.

If the patient is not in capacity to understand and/or express his/her consent, the informed consent will be 


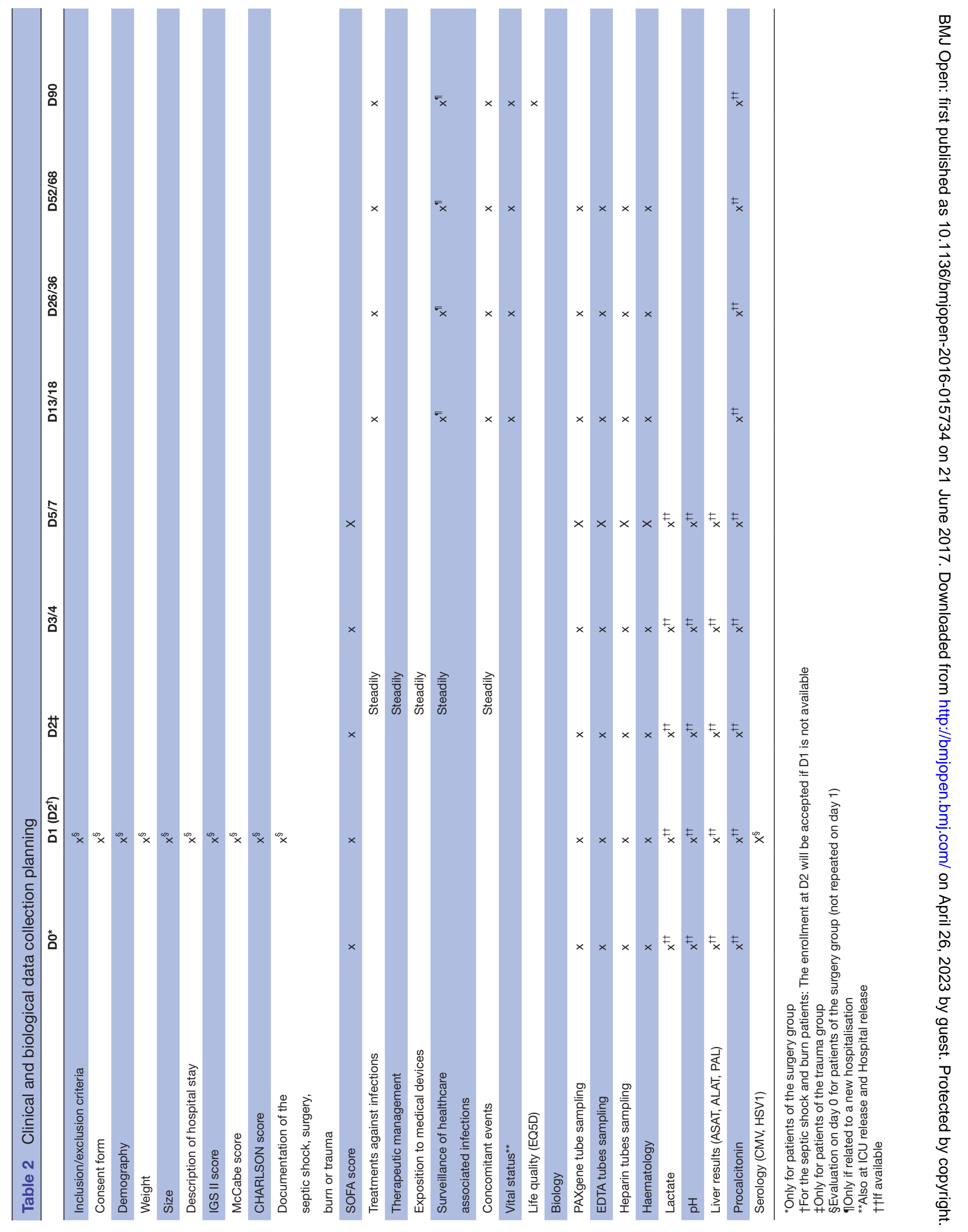


obtained from a next of kin. In the event that only the informed consent of a third party has been sought at the time of inclusion, the patients should be informed as soon as possible of their participation in this study and be asked to give their own consent to continue the study.

If the next of kin is not present and not available by phone, the patient may be included in emergency situation. The investigator will be required to record all steps for calling the next of kin in the medical record (contact attempts with date, time and phone number) and justify patient inclusion in medical emergencies in accordance with French legislation. The written consent of the next of kin and the patient should be obtained as soon as the person is available and as soon as the patient's clinical condition allows. The consent form contains the possibility to refuse the storage of samples after the end of the study.

\section{Safety of participants}

This study includes no serious foreseeable risk to the health of the persons involved. The only potential risk is related to blood sample collection (maximum $192 \mathrm{~mL}$ collected over all time points - 2 months). However, this aspect of nursing is part of daily practice. Blood samples will be taken under the same conditions of safety as currently used for common diagnostic tests.

\section{Study management}

The study is managed by BIOASTER and a dedicated team composed from members of all the consortium partners. The promoter of the study is the Hospices Civils de Lyon. The principal investigator is Dr Thomas Rimmelé.

\section{Data management}

\section{Clinical data}

For each patient, an electronic case report form including socio-demographic, clinical and para-clinical information will be completed by clinical research assistants (table 2): adescription of the hospital stay, the documentation on the type of injury (surgery, burn, trauma or septic shock) and the severity as defined by the ASA classification, SOFA score ${ }^{30}$ and SAPSII score ${ }^{30}$ In addition, we will collect routine laboratory results about the CMV, HSV1 serology and complete blood count. Moreover, we will document if there is any specific treatments administered to the patient, such as antibiotics, exposure to invasive medical devices and secondary infections. All data will be transferred to a TranSMART ${ }^{30}$ database following curation for data exploration and analysis.

\section{Duration of the study}

The study is planned to run for 30 months, starting December 2015. The expected end date for recruitment is June 2018. Some biomarkers will be quantified by batch analysis, at the end of the study. Primary data analysis is expected to be completed with subsequent dissemination of results by December 2018 .
${ }^{2}$ EA7426 "Pathophysiology of Injury-induced immunosuppression", Université Claude Bernard Lyon 1 - Hospices Civils de Lyon - bioMérieux, Lyon, France ${ }^{3}$ Immunology Laboratory, Hospices Civils de Lyon - Université Claude Bernard Lyon 1, Lyon, France

${ }^{4}$ Anesthesiology and Critical Care Medicine, Hospices Civils de Lyon - Université Claude Bernard Lyon 1, Lyon, France

${ }^{5}$ Medical Diagnostic Discovery Department (MD3), bioMérieux, Marcy-l'Étoile, France

${ }^{6} \mathrm{R} \& D$, Sanofi Aventis, Chilly-Mazarin, France

${ }^{7}$ Sanofi-Pasteur SA, Lyon, France

${ }^{8}$ ESPCI Paris, PSL Research University, Paris, France

${ }^{13}$ GlaxoSmithKline, Collegeville, PA, USA

Correction notice This paper has been amended since it was published Online First. Owing to a scripting error, some of the publisher names in the references were replaced with 'BMJ Publishing Group'. This only affected the full text version, not the PDF. We have since corrected these errors and the correct publishers have been inserted into the references.

Acknowledgements The project is funded by a consortium: bioMérieux, SANOFI, GlaxoSmithKline, Ecole Supérieure de Physique Chimie Industrielles de la ville the Paris - PSL Research University, the University Hospital Hospices Civils de Lyon and the microbiology technological institute BIOASTER. The project is financially supported in part by public funding through BIOASTER and Hospices Civils de Lyon. The project will be audited annually by the French National Research Agency (“Investissement d'Avenir" program; grant no ANR7107AIRT703).

Collaborators For Hospices Civils de Lyon: Asma BEN AMOR, André BOIBIEUX, Julien DAVIDSON, Laure FAYOLLE-PIVOT, Charline GENIN, Arnaud GREGOIRE, Alain LEPAPE, Anne Claire LUKASZEWICZ, Guillaume MARCOTTE, Delphine MAUCORT-BOULCH, Boris MEUNIER, Guillaume MONNERET, Nathalie PANEL, Thomas RIMMELE, Hélène VALLIN and Fabienne VENET. For bioMérieux: Sophie BLEIN, Karen BRENGEL-PESCE, Elisabeth CERRATO, Valérie CHEYNET, Emmanuelle GALLET-GORIUS, Audrey GUICHARD, François MALLET, Virginie MOUCADEL, Marine MOMMERT, Guy ORIOL, Alexandre PACHOT, Claire SCHREVEL, Olivier TABONE, Julien TEXTORIS and Javier YUGUEROS MARCOS. For BIOASTER: Jérémie BECKER, Frédéric BEQUET, Yacine BOUNAB, Nathalie GARCON, Irène GORSE, Cyril GUYARD, Fabien LAVOCAT, Philippe LEISSNER, Karen LOUIS, Maxime MISTRETTA, Yoann MOUSCAZ, Laura NOAILLES, Magali PERRET, Frédéric REYNIER, Cindy RIFFAUD, Mary Luz ROL, Nicolas SAPAY, Trang TRAN and Christophe VEDRINE. For Sanofi: Nicolas BURDIN, Christophe CARRE, Pierre CORTEZ, Aymeric DE MONFORT, Karine FLORIN, Laurent FRAISSE, Isabelle FUGIER, Sandrine PAYRARD, Annick PELERAUX and Laurence QUEMENEUR. For ESPCI Paris: Andrew GRIFFITHS and Stephanie TOETSCH. For GSK: Theresa ASHTON, Peter GOUGH, Scott BERGER, Lionel TAN, lain GILLESPIE and David GARDINER.

Contributors All authors (M-LR, FV, TR, VM, PC, LQ, DG, AG, AP, JT and GM) fulfilled ICMJE guidelines and provided substantial contributions to conception, design and acquisition of data; drafted and revised critically the manuscript; and approved the final version of the manuscript.

Competing interests AP, JT and VM are employees of bioMérieux SA, an in vitro diagnostic company. PC, LQ and DG are employees of Sanofi-Aventis R\&D, SanofiPasteur SA and GlaxoSmithKline, three pharmaceutical companies.

Ethics approval Comit de Protection des Personnes Lyon Sud-Est 2.

Provenance and peer review Not commissioned; externally peer reviewed.

Data sharing statement Results will be disseminated through presentations at scientific meetings and publications in peer-reviewed journals. New markers and immune functional tests will be evaluated for the diagnostic immune deficiency and may be patentable.

Open Access This is an Open Access article distributed in accordance with the Creative Commons Attribution Non Commercial (CC BY-NC 4.0) license, which permits others to distribute, remix, adapt, build upon this work non-commercially, and license their derivative works on different terms, provided the original work is properly cited and the use is non-commercial. See: http://creativecommons.org/ licenses/by-nc/4.0/

(c) Article author(s) (or their employer(s) unless otherwise stated in the text of the article) 2017. All rights reserved. No commercial use is permitted unless otherwise expressly granted. 


\section{REFERENCES}

1. Jawad I, Lukšić I, Rafnsson SB. Assessing available information on the burden of sepsis: global estimates of incidence, prevalence and mortality. J Glob Health 2012;2:4.

2. Vincent JL, Marshall JC, Namendys-Silva SA, et al. Assessment of the worldwide burden of critical illness: the Intensive Care Over Nations (ICON) audit. Lancet Respir Med 2014;2:380-6.

3. Walkey AJ, Lagu T, Lindenauer PK. Trends in sepsis and infection sources in the United States. A population-based study. Ann Am Thorac Soc 2015;12:216-20.

4. Seymour CW, Rea TD, Kahn JM, et al. Severe sepsis in pre-hospital emergency care: analysis of incidence, care, and outcome. Am J Respir Crit Care Med 2012;186:1264-71.

5. Singer M, Deutschman CS, Seymour CW, et al. The Third International Consensus Definitions for Sepsis and Septic shock (Sepsis-3). JAMA 2016;315:801.

6. Angus DC, van der Poll T, Sepsis S, et al. Severe sepsis and septic shock. N Engl J Med 2013;369:840-51.

7. Cohen J, Vincent JL, Adhikari NK, et al. Sepsis: a roadmap for future research. Lancet Infect Dis 2015;15:581-614.

8. Hotchkiss RS, Monneret G, Payen D. Sepsis-induced immunosuppression: from cellular dysfunctions to immunotherapy. Nat Rev Immunol 2013;13:862-74.

9. Hotchkiss RS, Monneret G, Payen D. Immunosuppression in sepsis: a novel understanding of the disorder and a new therapeutic approach. Lancet Infect Dis 2013;13:260-8.

10. Delsing CE, Gresnigt MS, Leentjens J, et al. Interferon-gamma as adjunctive immunotherapy for invasive fungal infections: a case series. BMC Infect Dis 2014;14:166.

11. Venet F, Lukaszewicz AC, Payen D, et al. Monitoring the immune response in sepsis: a rational approach to administration of immunoadjuvant therapies. Curr Opin Immunol 2013;25:477-83.

12. Meisel C, Schefold JC, Pschowski R, et al. Granulocytemacrophage colony-stimulating factor to reverse sepsis-associated immunosuppression: a double-blind, randomized, placebo-controlled multicenter trial. Am J Respir Crit Care Med 2009;180:640-8.

13. Gentile LF, Cuenca AG, Efron PA, et al. Persistent inflammation and immunosuppression: a common syndrome and new horizon for surgical intensive care. J Trauma Acute Care Surg 2012;72:1491-501.

14. Angele MK, Chaudry IH. Surgical trauma and immunosuppression: pathophysiology and potential immunomodulatory approaches. Langenbecks Arch Surg 2005;390:333-41.

15. Kimura F, Shimizu H, Yoshidome $H$, et al. Immunosuppression following surgical and traumatic injury. Surg Today 2010;40:793-808.

16. Timmermans K, Kox M, Vaneker M, et al. Plasma levels of dangerassociated molecular patterns are associated with immune suppression in trauma patients. Intensive Care Med 2016;42:551-61.
17. Gossez M, Malcus C, Demaret J, et al. Evaluation of a novel automated volumetric flow cytometer for absolute CD4+ T lymphocyte quantitation. Cytometry B Clin Cytom 2016:n/a.

18. http://www.clinicaltrial.gov/.

19. Duffy D, Rouilly V, Libri V, et al. Functional analysis via standardized whole-blood stimulation systems defines the boundaries of a healthy immune response to complex stimuli. Immunity 2014;40:436-50.

20. Poujol F, Monneret G, Friggeri A, et al. Flow cytometric evaluation of lymphocyte transformation test based on 5-ethynyl-2'deoxyuridine incorporation as a clinical alternative to tritiated thymidine uptake measurement. J Immunol Methods 2014;415:71-9.

21. Baker SP, O'Neill B, Haddon W, et al. The injury severity score: a method for describing patients with multiple injuries and evaluating emergency care. J Trauma 1974;14:187-96.

22. European Centre for Disease Prevention and Control. Point prevalence survey of healthcareassociated infections and antimicrobial use in european acute care hospitals: ECDC, 2012.

23. van Vught LA, Klein Klouwenberg PM, Spitoni $C$, et al. Incidence, risk factors, and attributable mortality of secondary infections in the intensive care unit after admission for sepsis. JAMA 2016;315:1469-79.

24. Demaret J, Venet F, Friggeri A, et al. Marked alterations of neutrophil functions during sepsis-induced immunosuppression. J Leukoc Biol 2015;98:1081-90.

25. Venet $\mathrm{F}$, Chung $\mathrm{CS}$, Kherouf $\mathrm{H}$, et al. Increased circulating regulatory T cells (CD4(+)CD25 (+)CD127 (-)) contribute to lymphocyte anergy in septic shock patients. Intensive Care Med 2009;35:678-86.

26. Döcke WD, Höflich C, Davis KA, et al. Monitoring temporary immunodepression by flow cytometric measurement of monocytic HLA-DR expression: a multicenter standardized study. Clin Chem 2005;51:2341-7.

27. Horowitz GL, Altaie S, Boyd JC, et al. CLSI C28-A3 defining, establishing and verifying reference intervals in the Clinical Laboratory Approved Guideline, 2008.

28. Fine JP, Gray RJ. A proportional hazards Model for the subdistribution of a competing risk. J Am Stat Assoc 1999;94:496-509.

29. Vincent JL, Moreno R, Takala J, et al. The SOFA (Sepsis-related organ Failure Assessment) score to describe organ dysfunction/ failure. On behalf of the Working Group on Sepsis-Related problems of the European Society of Intensive Care Medicine. Intensive Care Med 1996;22:707-10.

30. Le Gall JR, Lemeshow S, Saulnier F. A New Simplified Acute Physiology Score (SAPS II) based on a European/North American Multicenter study. JAMA 1993;270:2957-63.

31. TranSMART. http://transmartfoundation.org/. 